\title{
ON A GENERAL CLASS OF SERIES OF THE FORM $\sum c_{n} g(x+n)^{*}$
}

BY

\author{
R. D. CARMICHAEL
}

\section{INTRODUCTION}

In several important investigations, beginning with two memoirs $\dagger$ by Poincaré in 1885 and 1886 respectively, functions have arisen which are analytic in a sector of the complex plane and in that sector have a determinate asymptotic character (in the sense of the definition of Poincaré). In these investigations it has been usual to represent the asymptotic character of such a function by means of a divergent power series and to find in addition a convergent expansion which puts in evidence the analytic character of the function in the sector. Often this convergent expansion is complicated in character.

Obviously, it is desirable, if possible, to have a convergent expansion which accomplishes the latter object and at the same time readily shows the asymptotic character of the function. Recently Nörlund $\ddagger$ has shown that functions which are characterized by being analytic in a half-plane and having an asymptotic character of a certain broad type are expansible in convergent factorial series and that these series do in fact readily show the asymptotic character of the functions represented by them. He has thus made manifest the fundamental importance of this class of series in investigating the properties of a function in the neighborhood of a singularity of a certain type.

Some months ago I observed that the factorial series is but an instance of a large classई of series of simple properties and suitable for the representation of functions which are defined throughout a half-plane and have certain types of singularities at infinity. These series are of the form

* Presented to the Society (at Columbus), December 31, 1915.

† A merican Journal of Mathematics, vol. 7 (1885), pp. 203-258; A c t a M a th e matica, vol. 8 (1886), pp. 295-344. Particular instances of such functions appeared earlier, notably in the work of Stirling and Cauchy (see Borel's Séries divergentes, p. 30).

$\ddagger$ A c t a M a th e matic a, vol. 37 (1914), pp. 327-387. Two other valuable contributions leading in the same general direction should be mentioned, namely, a paper by Horn in $\mathrm{Mathematische} \mathrm{Annalen,} \mathrm{vol.} 71$ (1912), pp. 510-532 and one by Watson in Rendiconti del Circolo Matematicodi Palermo, vol. 34·(1912), pp. 41-88. class.

$\S$ In $\$ 6$ it is seen incidentally that the power series is also essentially contained in this 


$$
\bar{\Omega}(x)=\sum_{n=0}^{\infty} c_{n} \frac{g(x+n)}{g(x)},
$$

where $g(x)$ is a given function of $x$ and the coefficients $c_{n}$ are independent of $x$. With these series I associate others of the form

$$
\Omega(x)=\sum_{n=0}^{\infty} c_{n} g(x+n) .
$$

More precise definitions are given at the beginning of $\S 1$. As will be seen from these definitions, the fundamental characteristic of $g(x)$ is its asymptotic behavior. A detailed study of the more important series included in these classes and especially of their suitability for the representation of certain types of functions is desirable and will doubtless have important consequences in the development of the theory of functions. Results of this character I expect to communicate to the Society in later contributions. From these latter the important connection of the series $\bar{\Omega}(x)$ with the function-theoretic considerations mentioned in the first two paragraphs of this introduction will become apparent. In the present memoir I lay the foundations of the general theory of these series. Among the results obtained there is a set which corresponds closely, in point of completeness, to those contained in Landau's valuable memoir* on the foundations of the theory of factorial series. In the derivation of these results I have found Landau's methods of constant value and have employed them freely.

After a definition in $\S 1$ of the classes $\Omega(x)$ and $\bar{\Omega}(x)$ of series in consideration I proceed in $\$ \S 2-4$ to the complete determination of the character of the regions of convergence and absolute convergence of the series and to a consideration of the character of their convergence in respect to uniformity. Certain auxiliary series (of the nature of generalized Dirichlet series) are introduced in $\$ 5$ and their properties are developed so far as they are needed for the determination in $\S 6$ of the precise regions of convergence and absolute convergence of a particular series $\Omega(x)$ or $\bar{\Omega}(x)$ in terms of its coefficients $c_{n}$. In $\$ 7$ further relations between the series $\Omega(x)$ and the auxiliary generalized Dirichlet series are developed and are then employed in showing the existence, in certain cases, of singularities of functions defined by the series $\Omega(x)$. In $\S 8$ I observe that a function can sometimes be expanded in a series $\Omega(x)$ or a series $\bar{\Omega}(x)$ dependent on a given function $g(x)$, in more than one way and indeed even in an unlimited number of ways; but if a certain broad addi-

\footnotetext{
* Sitzungsberichtedermathematisch-physikalischen Klasse der königlichen Bayerischen Akademie der Wissenschaften z u M ü n c h e n, vol. 36 (1906): 151-218. This paper is valuable both for its elegant exposition of the theory of factorial series and for its new methods and results and for the historical remarks contained in it.
} 
tional condition is placed on the function $g(x)$ it is shown that the expansion of a function in the series $\Omega(x)$ or in the series $\bar{\Omega}(x)$ can be effected in no more than one way.

\section{Definitions and general notions}

Let $g(x)$ be a singie-valued analytic function of the complex variable $x$ having the asymptotic character

$$
g(x) \sim x^{P(x)} e^{Q(x)}\left(1+\frac{a_{1}}{x}+\frac{a_{2}}{x^{2}}+\cdots\right)
$$

valid for $x$ approaching infinity in a positive sense along any line whatever parallel to the axis of reals, the functions $P(x)$ and $Q(x)$ being polynomials which we write in the form

$$
\begin{aligned}
& P(x)=\mu_{0}+\mu_{1} x+\mu_{2} x^{2}+\cdots+\mu_{k} x^{k} \quad\left(\mu_{k} \neq 0 \text { if } k>0\right), \\
& Q(x)=\alpha_{0}+\alpha_{1} x+\alpha_{2} x^{2}+\cdots+\alpha_{m} x^{m} \quad\left(\alpha_{m} \neq 0 \text { if } m>0\right) .
\end{aligned}
$$

In case $k=0$ we assume that $m$ is greater than unity in order to avoid cases which are unimportant so far as our present objects are concerned.* By $r^{t}$ we mean $e^{t \log r}$ where the principal determination of $\log r$ is taken.

The asymptotic relation (1) is to be understood as an abbreviation for the infinite sequence of limits

$$
\begin{aligned}
\lim x^{s}\left\{g(x) x^{-P(x)} e^{-Q(x)}-\left(1+\frac{a_{1}}{x}+\cdots+\frac{a_{s}}{x^{s}}\right)\right\} & =0 \\
& (s=0,1,2, \cdots),
\end{aligned}
$$

the limit being taken for $x$ approaching infinity in a positive sense along any line whatever parallel to the axis of reals. $\dagger$

By means of any function $g(x)$ possessing the properties just indicated we introduce the series $\Omega(x)$ defined by the relation

$$
\Omega(x)=\sum_{n=0}^{\infty} c_{n} g(x+n)
$$

where the coefficients $c_{0}, c_{1}, c_{2}, \cdots$ are independent of $x$. For the applications it will often be convenient to consider the series $\bar{\Omega}(x)$ which we write in the form

* The case excluded not only is unimportant for our purposes but it also requires a different method of treatment.

$\dagger$ The reader, in the course of perusing the paper, will readily observe that a weaker restriction than that indicated by the infinite set of limits (2) will suffice for the derivation of many of the results. In particular, throughout $\$ \S 2-4$ (in which the character of the regions of convergence and absolute convergence of $\Omega(x)$ is determined) the limits for $s=0$ and $s=1$ will suffice; and that for $s=0$ alone will suffice unless simultaneously $k=1$ and $m=0$ or 1 . 


$$
\bar{\Omega}(x) \equiv \frac{\Omega(x)}{g(x)}=\sum_{n=0}^{\infty} c_{n} \frac{g(x+n)}{g(x)} .
$$

A value of $x$ for which any one of the functions $g(x), g(x+1), g(x+2)$, $\cdots[g(x+1) / g(x), g(x+2) / g(x), \cdots]$ has a singularity will be called an exceptional value or an exceptional point for the series $\Omega(x)[\bar{\Omega}(x)]$. Other values of $x$ will be called non-exceptional. In order to avoid cases without value for our purposes we shall assume that $g(x)$ is of such character that there is a (two-dimensional) region in the $x$-plane containing no exceptional points for the series.

That a large and important class of series $\Omega(x)$ and $\bar{\Omega}(x)$ exist in accordance with the foregoing definition is readily apparent from the following considerations.

In the first place there exists an extensive and easily accessible class of functions $g(x)$. In particular, such functions are introduced to our notice by the linear homogeneous differential equation

$$
P_{0} \frac{d^{n} y}{d x^{n}}+P_{1} \frac{d^{n-1} y}{d x^{n-1}}+\cdots+P_{n} y=0,
$$

in which the coefficients $P_{0}, P_{1}, \cdots, P_{n}$ are polynomials in $x$ subject to the condition that their degrees do not constantly decrease as one passes in order from the first to the last polynomial in the sequence. Poincare* ${ }^{*}$ has in fact shown that a solution $y(x)$ of such an equation is asymptotic to an expression of the form of the second member of (1) when the polynomial $P(x)$ is taken to be a constant and $x$ approaches infinity in an appropriate direction. Hence a constant $c$ exists such that $y(c x)$ is a function having the property specified above for $g(x)$.

A still more effective (because simpler) class of functions $g(x)$ is afforded by linear homogeneous difference equations whose coefficients have at infinity the character of rational functions and whose associated characteristic algebraic equation has its roots different from each other and from zero. Such an equation has a fundamental set of solutions $\dagger$ each function of which is of the character specified above for $g(x)$, the polynomials $P(x)$ and $Q(x)$ in this case being of the first degree. The series $\bar{\Omega}(x)$ has been treated in detail (see Landau, l. c.) for the two special values $f g(x)=\Gamma(x)$ and $g(x)=1 / \Gamma(x)$ belonging to this sub-class. The latter value of $g(x)$ gives rise to the simple factorial series

*A c ta M a them atic a, vol. 8 (1886), pp. 295-344.

$\dagger$ Birkhoff, these Tran s a c t i o n s, vol. 12 (1911): 242-284. See also an earlier paper of mine in the same volume.

$\ddagger$ For the first case mentioned a simple transformation is necessary in order to throw the series $\bar{\Omega}(x)$ into the form treated by Landau. 


$$
\bar{\Omega}(x)=c_{0}+\sum_{n=0}^{\infty} \frac{c_{n+1}}{x(x+1) \cdots(x+n)},
$$

the impcrtance of which has already been indicated in the introduction.*

Among the important special types of series of the class $\bar{\Omega}(x)$ introduced by means of difference equations is the important generalization of factorial series afforded by the series

$$
\bar{\Omega}(x)=c_{0}+\sum_{n=0}^{\infty} c_{n+1} A(x) A(x+1) \cdots A(x+n),
$$

where $A(x)$ is any rational function of $x$ having a zero value for $x=\infty$. It arises through taking for $g(x)$ an appropriate solution (in fact, the socalled first principal solution) of the equation $f(x+1)=A(x) f(x)$; for from this equation we have readily

$$
f(x+n+1)=A(x+n) A(x+n-1) \cdots A(x+1) A(x) f(x) .
$$

In determining the character of the region of convergence of $\Omega(x)$ (and consequently of $\bar{\Omega}(x)$ ) we have need for two lemmas $\dagger$ which it is convenient to state here as follows:

Lemma I. Let $u_{1}+u_{2}+u_{3}+\cdots$ be a convergent series and let $v_{1}, v_{2}$, $v_{3}, \cdots$ be an infinite sequence of numbers such that the series

is convergent. Then the series

$$
\sum_{n=1}^{\infty}\left|v_{n}-v_{n+1}\right|
$$

is convergent.

$$
u_{1} v_{1}+u_{2} v_{2}+u_{3} v_{3}+\cdots
$$

LEMMA II. Let $u_{1}+u_{2}+u_{3}+\cdots$ be a convergent series of constant terms and let $v_{1}, v_{2}, v_{3}, \cdots$ be an infinite sequence of functions of the complex variable $x$ analytic in a given closed domain $D$ and such that the series

$$
\sum_{n=1}^{\infty}\left|v_{n}-v_{n+1}\right|
$$

converges uniformly in $D$. Then the series

$$
u_{1} v_{1}+u_{2} v_{2}+u_{3} v_{3}+\cdots
$$

converges uniformly in $D$.

* The definitions of the series $\Omega(x)$ and $\bar{\Omega}(x)$ might be extended to the case in which the series in (1) proceeds in certain descending fractional powers of $x$. The resulting series are amenable to the general methods of this paper. Again, suitable functions $g(x)$ for these series are readily afforded by the solutions of linear differential and difference equations.

† These lemmas have been employed by several writers. The reader will conveniently find proofs of them (in a generalized form) in the Bulletin of the American Mathematical S o ciety, vol. 20, pp. 225-233. Historical remarks concerning their previous use will be found in Landau's memoir (already cited). 


\section{Character of the Region of convergence}

Suppose that the series $\Omega(x)$ converges for a given value $x_{0}$ of $x$ which is non-exceptional for $\Omega(x)$. We shall find conditions on a value $x_{1}$ of $x$ which are sufficient to ensure that $\Omega(x)$ converges for $x=x_{1}, x_{1}$ itself being a nonexceptional value for $\Omega(x)$. For this purpose we may employ Lemma I, taking

$$
u_{n}=c_{n} g\left(x_{0}+n\right), \quad v_{n}=\frac{g\left(x_{1}+n\right)}{g\left(x_{0}+n\right)}, \quad n \geqq l ;
$$

here the integer $l$ is chosen so that $g\left(x_{0}+n\right) \neq 0$ if $n \geqq l$. Then $\Omega\left(x_{1}\right)$ will converge* provided the series

is convergent.

$$
\sum_{n=l}^{\infty}\left|v_{n}-v_{n+1}\right|
$$

For determining when the series (6) converges we make use of the asymptotic character of $v_{n}$ with respect to $n$. To obtain a formula for this it is convenient to write first the formula for the asymptotic character of $g(x+n)$ with respect to $n$ when $x$ is held fixed. We have

$$
g(x+n) \sim e^{p_{n}(x) \log n+q_{n}(x)} s_{n}(1)
$$

where $s_{n}(\alpha)$ denotes a series in non-negative integral powers of $1 / n$ with constant term $\alpha$ and $p_{n}(x)$ and $q_{n}(x)$ are quantities which may be written in the form

$$
\begin{aligned}
p_{n}(x)=\nu_{0 n}+\nu_{1 n} x+\nu_{2 n} x^{2}+\cdots+ & +\nu_{k n} x^{k}, \\
q_{n}(x)=\beta_{0 n}+\beta_{1 n} x+\beta_{2 n} x^{2}+\cdots & +\beta_{m n} x^{m} \\
& +\gamma_{1 n} x+\gamma_{2 n} x^{2}+\cdots+\gamma_{k n} x^{k} .
\end{aligned}
$$

Here the quantities $\nu, \beta, \gamma$ have the values

$$
\begin{aligned}
\nu_{s n} & =\sum_{t=s}^{k}\left(\begin{array}{l}
t \\
s
\end{array}\right) \mu_{t} n^{t-s}, \quad s=0,1, \cdots, k \\
\beta_{s n} & =\sum_{t=s}^{m}\left(\begin{array}{l}
t \\
s
\end{array}\right) \alpha_{t} n^{t-s}, \quad s=0,1, \cdots, m \\
\gamma_{s n} & =\sum_{t=0}^{k} \mu_{t} n^{t-s}\left[\left(\begin{array}{c}
t \\
s-1
\end{array}\right)-\frac{1}{2}\left(\begin{array}{c}
t \\
s-2
\end{array}\right)+\frac{1}{3}\left(\begin{array}{c}
t \\
s-3
\end{array}\right)-\cdots\right]
\end{aligned}
$$

From (7) it follows that

$$
(s=1,2, \cdots, k) \text {. }
$$

$$
v_{n} \sim e^{\log n \cdot\left\{p_{n}\left(x_{1}\right)-p_{n}\left(x_{0}\right)\right\}+\left\{q_{n}\left(x_{1}\right)-q_{n}\left(x_{0}\right)\right\}} s_{n}(1) .
$$

* We employ the statement $\Omega\left(x_{1}\right)$ converges as an abbreviation for the statement the series $\Omega(x)$ converges for the value $x_{1}$ of $x$. 
By means of this relation we determine conditions under which series (6) is convergent. We see that $p_{n}\left(x_{1}\right)-p_{n}\left(x_{0}\right)$ is a polynomial in $n$ of degree $k-1$ and that $q_{n}\left(x_{1}\right)-q_{n}\left(x_{0}\right)$ is a polynomial in $n$ and that its degree is $m-1$ in case $m$ is greater than $k$. (In case $k$ or $m$ is zero the corresponding difference function is zero.) There is obviously a separation of cases according as $k$ is or is not less than $m$.

Suppose first that $k \geqq m$. Then it is clear that the dominating term in the exponent of the second member of (10), when this exponent is expressed as a polynomial in $n$ and $\log n$, is

$$
k \mu_{k}\left(x_{1}-x_{0}\right) n^{k-1} \log n .
$$

Then it is easy to see that series (6) converges if $R\left\{\mu_{k}\left(x_{1}-x_{0}\right)\right\}^{*}$ is less than zero, since clearly it is dominated term by term by a convergent series. This is obvious in case $k>1$. If $k=1$ we have

$$
v_{n} \sim n^{\mu_{1}\left(x_{1}-x_{0}\right)}\left(t_{0}+\frac{t_{1}}{n}+\cdots\right),
$$

where $t_{0}, t_{1}, \cdots$ are determinate constants. Then

$$
v_{n}-v_{n+1} \sim n^{\mu_{1}\left(x_{1}-x_{0}\right)-1}\left(t_{0} \mu_{1}\left(x_{0}-x_{1}\right)+\frac{\xi}{n}+\cdots\right),
$$

where $\xi$ is a determinate constant. From this it follows in this case also that series (6) converges if $R\left\{\mu_{k}\left(x_{1}-x_{0}\right)\right\}$ is negative.

/ Next suppose that $k<m$. Then $m \geqq 2$, since by hypothesis $m>1$ if $k=0$. The dominating term in the exponent in the second member of (10), when this exponent is expressed as a polynomial in $n$ and $\log n$, is

$$
m \alpha_{m}\left(x_{1}-x_{0}\right) n^{m-1} \text {. }
$$

Hence, in this case series (6) converges when $R\left\{\alpha_{m}\left(x_{1}-x_{0}\right)\right\}$ is negative.

From Lemma $I$ it follows that the convergence of (6) is sufficient to ensure the convergence of $\Omega\left(x_{1}\right)$. Hence we are led to the following theorem:

Theorem I. Let $x_{0}$ and $x_{1}$ be two values of $x$ which are non-exceptional for the series $\Omega(x)$ and suppose that $\Omega\left(x_{0}\right)$ converges. Then

(1) in case $k \geqq m, \Omega\left(x_{1}\right)$ converges if $R\left(\mu_{k} x_{1}\right)<R\left(\mu_{k} x_{0}\right)$;

(2) in case $k<m, \Omega\left(x_{1}\right)$ converges if $R\left(\alpha_{m} x_{1}\right)<R\left(\alpha_{m} x_{0}\right)$.

By the region $C$ of convergence of the series $\Omega(x)$ we shall mean a region such that $\Omega(x)$ converges for every non-exceptional value of $x$ in the interior of $C$ and diverges for every non-exceptional value of $x$ exterior to $C$. We shall likewise call $C$ the region of convergence of the series $\bar{\Omega}(x)$ since ob-

* The symbol $R(z)$ is used to denote the real part of $z$. 
viously $\Omega(x)$ and $\bar{\Omega}(x)$ both converge or both diverge for a given non-exceptional value of $x$ for which $g(x)$ is finite and different from zero.

By means of the foregoing theorem it is easy to determine the nature of the region of convergence of $\Omega(x)$. Through examples it may be shown in the first place that particular series $\Omega(x)$ exist which diverge for every non-exceptional value of $x$ and that there are other particular series $\Omega(x)$ which converge for every non-exceptional value of $x$. In fact such examples are readily afforded by the theory of factorial series. We shall assume for the moment that we are dealing with a series $\Omega(x)$ which belongs neither to the class of everywhere convergent series nor to the class of everywhere divergent series. Let $\sigma$ denote $\alpha_{m}$ or $\mu_{k}$ according as $m$ is or is not greater than $k$. Consider the real numbers $R(\sigma x)$ for varying values of $x$ which are non-exceptional for $\Omega(x)$ and divide them into two classes $A$ and $B$ according as $x$ is a point of convergence or a point of divergence for $\Omega(x)$. From Theorem $\mathrm{I}$ it follows that no $B$ is less than any $A$. Then it is clear that a number $\lambda$ exists such that $\Omega(x)$ converges [diverges] for every non-exceptional value of $x$ such that $R(\sigma x)<\lambda[R(\sigma x)>\lambda]$. Such a number $\lambda$ we shall call a convergence number for $\Omega(x)$. It may conveniently be said that $-\infty[+\infty]$ is a convergence number for $\Omega(x)$ when $\Omega(x)$ diverges [converges] for all non-exceptional values of $x$.

It is clear that a series $\Omega(x)$ has a unique convergence number $\lambda$ if the non-exceptional values of $x$ are such that the numbers $R(\sigma x)$ form a dense set on the whole of the axis of reals from $-\infty$ to $+\infty$; and this in fact is the case in the applications of the theory which we shall have occasion to make. When there is but one convergence number $\lambda$ we shall call it the convergence number for $\Omega(x)$. If there is more than one convergence number $\lambda$ then for the convergence number for $\Omega(x)$ we shall take the least convergence number or the greatest lower bound to the convergence numbers in case a least one does not exist.

If $\lambda$ is the convergence number for $\Omega(x)$, then the equation $R(\sigma x)=\lambda$ represents a straight line which is clearly the boundary of the region of convergence of $\Omega(x)$, this region being that for which $R(\sigma x)<\lambda$.

The results just derived may be stated in the following theorem:

TheOREM II. If $\sigma$ denotes $\alpha_{m}$ or $\mu_{k}$ according as $m$ is or is not greater than $k$, then there exists a unique real number $\lambda$ such that the region of convergence of the series $\Omega(x)[\bar{\Omega}(x)]$ is bounded by the straight line $R(\sigma x)=\lambda$ and lies on that side of this line for which $R(\sigma x)<\lambda$.

For particular functions $g(x)$ the series $\Omega(x)$ may converge everywhere on the boundary line $l$ of its region of convergence, or it may converge nowhere on $l$, or it may converge at some points on $l$ and diverge at others, as one may see readily from the special case of factorial series; and these possibilities 
may severally be realized through the character of the coefficients $c_{n}$. But there are certain general classes of cases in which the question as to convergence on $l$ is answered (except for a finite number of points) by the character of the function $g(x)$ itself. For the discussion of these it is convenient to distinguish four cases.

Suppose first that $k$ is greater than either 1 or $m$, or that $k=m>1$ and $\mu_{k}$ and $\alpha_{k}$ lie on the same straight line through the point zero in the complex plane. Let us consider the question of convergence of series (6) when

$$
R\left(\mu_{k} x_{1}\right)=R\left(\mu_{k} x_{0}\right) .
$$

This depends on the asymptotic character of $v_{n}$ as given in (10). It is easy to see that (6) converges provided that

$$
R\left\{\frac{1}{2} k \mu_{k}\left(x_{1}^{2}-x_{0}^{2}\right)+\mu_{k-1}\left(x_{1}-x_{0}\right)\right\}<0 .
$$

By an argument similar to that by which Theorem II was proved it may now be shown that if $\Omega(x)$ converges for any non-exceptional value $x_{0}$ of $x$ on the line $l$ then there exists a unique real number $\lambda_{1}$ such that $\Omega\left(x_{1}\right)$ converges if $R_{1}<\lambda_{1}$ and diverges if $R_{1}>\lambda_{1}, R_{1}$ being defined by the relation

$$
R_{1}=R\left\{\frac{1}{2} k \mu_{k} x_{1}^{2}+\mu_{k-1} x_{1}\right\}
$$

and $x_{1}$ being a non-exceptional value of $x$. In this case the only non-exceptional points $x_{1}$ at which the question of convergence of $\Omega(x)$ is unanswered are those for which we have simultaneously $R_{1}=\lambda_{1}$ and $R\left(\mu_{k} x_{1}\right)=\lambda$; and clearly these are at most two in number.

If $k=m>1$ and $\mu_{k}$ and $\alpha_{k}$ do not lie on the same straight line through the point 0 in the complex plane it may be shown in a similar manner that if $\Omega(x)$ converges for a non-exceptional value $x_{0}$ on the line $l$ then a unique real number $\lambda_{1}$ exists such that $\Omega\left(x_{1}\right)$, where $x_{1}$ is non-exceptional, converges or diverges according as $R\left\{x_{1}\left(\mu_{k}+\alpha_{k}\right)\right\}$ is less than $\lambda_{1}$ or greater than $\lambda_{1}$. In this case the only non-exceptional point $x_{1}$ at which the question of convergence of $\Omega\left(x_{1}\right)$ is unanswered is that for which we have simultaneously

$$
R\left(\mu_{k} x_{1}\right)=\lambda, \quad R\left\{x_{1}\left(\mu_{k}+\alpha_{k}\right)\right\}=\lambda_{1} .
$$

We suppose next that $m=k+1$ and that $\mu_{k}$ and $\alpha_{k+1}$ do not lie on the same straight line through the point 0 of the complex plane. Then $k \geqq 1$ since $m>1$ if $k=0$. Proceeding as before we find that if $\Omega(x)$ converges for a non-exceptional value $x_{0}$ on the line $l$ then a unique real number $\lambda_{1}$ exists such that $\Omega\left(x_{1}\right)$, where $x_{1}$ is non-exceptional, converges or diverges according as $R\left(\mu_{k} x_{1}\right)$ is less than $\lambda_{1}$ or is greater than $\lambda_{1}$. In this case the only nonexceptional point $x_{1}$ at which the question of convergence of $\Omega\left(x_{1}\right)$ is unanswered is that for which we have simultaneously 


$$
R\left(\alpha_{k+1} x_{1}\right)=\lambda, \quad R\left(\mu_{k} x_{1}\right)=\lambda_{1} .
$$

Finally, suppose that $m>k+1$, or that $m=k+1$ and $\mu_{k}$ and $\alpha_{k+1}$ lie on the same straight line through the point 0 of the complex plane. In this case we find that if $\Omega(x)$ converges for a non-exceptional value $x_{0}$ of $x$ on the line $l$ then a unique real number $\lambda_{1}$ exists such that $\Omega\left(x_{1}\right)$ converges if $R_{1}<\lambda_{1}$ and diverges if $R_{1}>\lambda_{1}, R_{1}$ being defined by the relation

$$
R_{1}=R\left(\frac{1}{2} m \alpha_{m} x_{1}^{2}+\alpha_{m-1} x_{1}\right)
$$

and $x_{1}$ being a non-exceptional value of $x$. In this case the only non-exceptional points $x_{1}$ at which the question of convergence of $\Omega\left(x_{1}\right)$ is unanswered are those for which we have simultaneously

$$
R_{1}=\lambda_{1}, \quad R\left(\alpha_{m} x_{1}\right)=\lambda .
$$

It is obvious that certain other cases, exceptional in character, may be similarly treated by the general method employed in the four preceding paragraphs.

\section{UNIFORM CONVERGENCE}

Turning now to the question of uniform convergence, we shall prove the following theorem:

THEOREM III. The series $\Omega(x)[\bar{\Omega}(x)]$ converges uniformly in any closed domain $D$ which lies within the region of convergence of $\Omega(x)[\bar{\Omega}(x)]$ and contains no points which are exceptional for this series or are limit points of points which are exceptional for the series.

It is sufficient to prove the theorem for the case of the series $\Omega(x)$; for the other series may obviously be treated in a similar manner.

From the character of $D$ as specified in the theorem it is clear that a nonexceptional point $x_{0}$ exists such that $\Omega\left(x_{0}\right)$ converges and $R\left(\sigma x_{0}\right)$ is less than $R\left(\sigma x_{1}\right)$ for every value of $x_{1}$ lying in $D, \sigma$ being used as before for $\alpha_{m}$ or $\mu_{k}$ according as $m$ is or is not greater than $k$. From Lemma II it follows that for the proof of Theorem III it is sufficient to show that series (6) converges uniformly for $x_{1}$ in $D$ and $x_{0}$ fixed as just indicated. For the proof of the latter we employ relation (10), which is valid for every value of $x_{1}$ in $D$. By its aid one may construct, in an obvious manner, a series of positive constant terms which is term by term greater than series (6) for every $x_{1}$ in $D$; whence, through use of a theorem of Weierstrass, it follows that (6), and hence $\Omega\left(x_{1}\right)$, is uniformly convergent in $D$.

As an immediate consequence of Theorem III we see that the sum of the series $\Omega(x)[\bar{\Omega}(x)]$ is a function $S(x)$ of $x$ which is analytic at every nonexceptional point which is in the interior of its region of convergence and is not a 
limit point of exceptional points, and the derivatives of $S(x)$ at every such point may be found by differentiating the series $\Omega(x)[\bar{\Omega}(x)]$ term by term.

\section{Character of the region of absolute convergence}

In order to determine the character of the region of absolute convergence of $\Omega(x)$ let us consider a non-exceptional point $x_{0}$ for which $\Omega\left(x_{0}\right)$ converges absolutely. Then it is obvious that $\Omega\left(x_{1}\right)$ will converge absolutely provided that $x_{1}$ is non-exceptional for $\Omega(x)$ and $\left|v_{n}\right|$ is bounded, $v_{n}$ being defined as before in terms of $x_{1}$ and $x_{0}$. Again we have the asymptotic relation (10). From this it follows readily that $\left|v_{n}\right|$ is bounded provided that

$$
R\left(\sigma x_{1}\right)<R\left(\sigma x_{0}\right) .
$$

Hence we have the following theorem:

Theorem IV. Let $\sigma$ denote $\alpha_{m}$ or $\mu_{k}$ according as $m$ is or is not greater than $k$. Let $x_{0}$ and $x_{1}$ be two non-exceptional values of $x$ for the series $\Omega(x)$ and suppose that $\Omega\left(x_{0}\right)$ converges absolutely. Then $\Omega\left(x_{1}\right)$ will converge absolutely if

$$
R\left(\sigma x_{1}\right)<R\left(\sigma x_{0}\right) .
$$

By the region $\Gamma$ of absolute convergence of the series $\Omega(x)$ we shall mean a region such that $\Omega(x)$ converges absolutely for every non-exceptional value of $x$ in the interior of $\Gamma$ and fails to converge absolutely for each non-exceptional value of $x$ exterior to $\Gamma$. We shall also call $\Gamma$ the region of absolute convergence for the series $\bar{\Omega}(x)$, since obviously $\Omega(x)$ and $\bar{\Omega}(x)$ both converge absolutely or both fail to converge absolutely for a given non-exceptional value of $x$ for which $g(x)$ is finite and different from 0 .

The nature of this region $\Gamma$ may readily be determined by means of Theorem IV. In fact, a discussion similar to that which leads up to Theorem II now yields the following result:

Theorem V. If $\sigma$ denotes $\alpha_{m}$ or $\mu_{k}$ according as $m$ is or is not greater than $k$, then there exists a unique real number $\mu$ such that the region of absolute convergence of the series $\Omega(x)[\bar{\Omega}(x)]$ is bounded by the straight line $R(\sigma x)=\mu$ and lies on that side of this line for which $R(\sigma x)<\mu$.

In certain general classes of cases the question concerning the absolute convergence of $\Omega(x)$ on the boundary line $l$ of absolute convergence (except at a finite number of points) depends on the character of $g(x)$. A treatment of this matter similar to that following Theorem II for the case of the boundary line of convergence of $\Omega(x)$ yields a set of conclusions precisely similar to those obtained for the latter problem. 


\section{Properties of a Certain Class of auXiliary series}

In view of the asymptotic form of $g(x+n)$ with respect to $n$ when $i$ is held fixed, as given in relation (7), it is natural to introduce* in connection with $\Omega(x)$ the generalized $\dagger$ Dirichlet series $\ddagger$

$$
D(x)=\sum_{n=1}^{\infty} c_{n} e^{\lambda_{1 n} x+\lambda_{2 n} x^{2}+\cdots+\lambda_{t n} x^{t}}
$$

where the functions $\lambda_{j n}$ of $n$, for $j=1,2, \cdots, t$, have the following properties:

(1) one at least of the functions $\lambda_{j n}$ approaches infinity as $n$ increases indefinitely;

(2) the limit $\lim _{n=\infty} \arg \lambda_{j n}$ exists for every $j$ and is finite;

(3) in case $t$ is greater than unity, one of the functions $\lambda_{j n}$, say $\lambda_{s n}$ (where $s$ is fixed), dominates all the others in such wise that

$$
\lim _{n=\infty} \frac{\lambda_{j n}}{\lambda_{s n}}=0, \quad j \neq s
$$

(4) in case more than one function of the set $\lambda_{j n}, j=1,2, \cdots, t$, is not identically zero the function $\lambda_{s n}$ is of such character that the series

$$
\sum_{n=1}^{\infty} e^{-\left|\lambda_{s n}\right| \eta}
$$

converges for every positive number $\eta$;

(5) in case $\lambda_{s n}$ is the only function of the set $\lambda_{j n}, j=1,2, \cdots, t$, which is not identically zero then $\lambda_{s n}$ either has the property named in (4) or arg $\lambda_{s n}$ is a constant.

We shall denote the value of the limits in (2) by $\theta_{j}, j=1,2, \cdots, t$, so that we have

$$
\theta_{j}=\lim _{n=\infty} \arg \lambda_{j n} \quad(j=1,2, \cdots, t) .
$$

Now let $x_{0}$ be a value of $x$ such that $D\left(x_{0}\right)$ converges and consider what conditions may be placed on $x_{1}$ so that $D\left(x_{1}\right)$ shall certainly converge. Em-

* Compare the special case $\Delta(x)$ of $D(x)$ introduced at the beginning of $\S 6$.

$\dagger$ The series commonly known as the generalized Dirichlet series is that special case of the series $D(x)$ in (11) for which $t=1$ and $\lambda_{1 n}$ is real. I have not been able to find a discussion of the more general series $D(x)$ defined in the text.

$\ddagger$ The properties of these generalized Dirichlet series are here developed only so far as they are needed in deriving the properties of the series $\Omega(x)$. It is clear that the theory is readily capable of further extension. Moreover, the generalization might be carried further in the direction indicated by the present treatment and yet the essential elegance of the fundamental theorems be retained. But our purpose here is to employ these series merely as a tool for investigating the properties of the series $\Omega(x)$ and $\bar{\Omega}(x)$; and the treatment is restricted accordingly. 
ploying Lemma I and proceeding as in the case of the corresponding problem for the series $\Omega(x)$ we see that $D\left(x_{1}\right)$ will converge provided that

$$
\sum_{n=1}^{\infty}\left|w_{n+1}-w_{n}\right|
$$

converges, where $w_{n}$ has the value

$$
w_{n}=e^{\lambda_{1 n}\left(x_{1}-x_{0}\right)+\lambda_{2 n}\left(x_{1}^{2}-x_{0}^{2}\right)+\cdots+\lambda_{t n}\left(x_{1}^{t}-x_{0} t\right)} .
$$

Now, let us write $\lambda_{s n}=\left|\lambda_{s n}\right| e^{i \theta_{s n}}$. Then, by hypothesis, $\theta_{s n}$ approaches the finite value $\theta_{s}$ as $n$ increases indefinitely. If we suppose that $x_{1}$ is subject to the condition

$$
R\left\{e^{i \theta_{s}}\left(x_{1}^{s}-x_{0}^{s}\right)\right\}<0
$$

it is clear that a positive constant $\eta$ and a positive integer $N$ exist such that

$$
\left|w_{n}\right|<e^{-\left|\lambda_{s n}\right| \eta}
$$

when $n$ is greater than $N$. From this and properties (4) and (5) of $\lambda_{s n}$ it follows that (12) certainly converges unless every function $\lambda_{j n}, j=1,2, \cdots, t$, except $\lambda_{s n}$, is identically equal to zero and $\lambda_{s n}$ is of constant modulus $\theta_{s}$. For this latter case the convergence of (12) may be shown in a different way. We now have

where

$$
w_{n+1}-w_{n}=e^{\lambda_{n+1} \tau}-e^{\lambda_{n} \tau},
$$

Hence

$$
\lambda_{n}=\left|\lambda_{s n}\right| \quad \text { and } \quad \tau=e^{i \theta_{s}}\left(x_{1}^{s}-x_{0}^{s}\right) \text {. }
$$

so that

$$
w_{n+1}-w_{n}=\frac{1}{\tau} \int_{\lambda_{n}}^{\lambda_{n+1}} e^{u \tau} d u ;
$$

$$
\left|w_{n+1}-w_{n}\right| \leqq \frac{1}{|\tau|} \int_{\lambda_{n}}^{\lambda_{n+1}} e^{u R(\tau)} d u .
$$

The series of which the $n$th term is the second member of this relation is obviously convergent since $R(\tau)<0$ on account of the condition on $x_{1}$ expressed in (13). Therefore, the series in (12) is convergent, as was to be proved.

From our lemma it follows now that $D\left(x_{1}\right)$ converges provided that $x_{1}$ satisfies inequality (13). This result may obviously be used as Theorem I was employed in $\S 2$ in the proof of Theorem II and with a corresponding result. Thus we are led to the following theorem:

Theorem VI. If $D\left(x_{0}\right)$ converges then $D\left(x_{1}\right)$ will converge if

$$
R\left(e^{i \theta_{0}} x_{1}^{s}\right)<R\left(e^{i \theta_{0}} x_{0}^{s}\right) .
$$

There exists a unique real number $\lambda$ such that the region of convergence of the 
series $D(x)$ is bounded by the curve* $R\left(e^{i \theta_{*}} x_{0}^{s}\right)=\lambda$ and lies on that side of this curve for which $R\left(e^{i \theta_{*}} x^{s}\right)<\lambda$.

Corresponding to Theorems IV and V of the preceding section, we have the following theorem which is readily proved:

Theorem VII. If $D\left(x_{0}\right)$ converges absolutely then $D\left(x_{1}\right)$ will converge absolutely if

$$
R\left(e^{i \theta_{s}} x_{1}^{s}\right)<R\left(e^{i \theta_{s}} x_{0}^{s}\right) .
$$

There exists a unique real number $\mu$ such that the region of absolute convergence of $D(x)$ is bounded by the curve $R\left(e^{i \theta_{0}} x^{s}\right)=\mu$ and lies on that side of this curve for which $R\left(e^{i \theta_{1}} x^{8}\right)<\mu$.

The question as to convergence of $D(x)$ for points on the boundary curves of convergence can be answered (except at a finite number of points), in a large range of cases, in terms of the properties of the functions $\lambda_{j n}, j=1,2$, $\cdots, t$; but we have no present use for these results and consequently they will not be stated.

Employing Lemma II and proceeding by a method in all respects similar to that used in the proof of Theorem III one may demonstrate the following theorem:

Theorem VIII. The series $D(x)$ converges uniformly in any closed domain $S$ lying within its region of convergence.

Consequently, the sum of the series is a function which is analytic within the region of convergence of the series.

We shall now prove the following theorem:

Theorem IX. The boundary curve of the region of convergence [absolute convergence] is the same for the two series $D(x)$ and $D_{s}(x)$, where

$$
D_{s}(x)=\sum_{n=1}^{\infty} c_{n} e^{\lambda_{\text {sn }} x^{s}} \text {. }
$$

It should be observed that the theorem says nothing about convergence on the boundary of the region of convergence [absolute convergence].

If $D(x)$ and $D_{s}(x)$ are not identical then at least two of the functions $\lambda_{1 n}, \cdots, \lambda_{l n}$ in (11) are not identically zero. Hence, so far as there is anything in the theorem to be proved, we have a $\lambda_{s n}$ possessing property (4). The proof of the theorem falls into two parts.

1. Let $\bar{x}$ be any point in the interior of the region of convergence of $D(x)$. We shall show that $\bar{x}$ is likewise in the interior of the region of convergence of $D_{s}(x)$.

If $R\left(e^{i \theta_{s}} x^{s}\right)=\lambda$ is the boundary of the region of convergence of $D(x)$, then $R\left(e^{i \theta_{s}} \bar{x}^{8}\right)<\lambda$. It is obvious thence that numbers $x_{0}$ and $x_{1}$ exist such that

\footnotetext{
* This curve is a straight line only if $s=1$.
} 


$$
R\left(e^{i \theta_{8}} \bar{x}^{s}\right)<R\left(e^{i \theta_{s}} x_{1}^{s}\right)<R\left(e^{i \theta_{d}} x_{0}^{s}\right)<\lambda .
$$

Then $x_{0}$ and $x_{1}$ are points of convergence of $D(x)$. If we apply Lemma I, taking for $u_{n}$ and $v_{n}$ the values

$$
u_{n}=c_{n} e^{\lambda_{1 n} x_{0}+\ldots+\lambda_{t n} x_{0} t}, \quad v_{n}=e^{\lambda_{m} \lambda_{1} 1^{t}-\left(\lambda_{1 n} x_{0}+\ldots+\lambda_{t n} x_{0} t\right)},
$$

we see that $D_{s}\left(x_{1}\right)$ converges if $\sum\left|v_{n}-v_{n+1}\right|$ converges. That the latter series converges follows at once from the fact that $\lambda_{s n}$ possesses property (4) and that a positive number $\eta$ and a positive integer $N$ exist such that

$$
\left|v_{n}\right|<e^{-\left|\lambda_{s n}\right| \eta}
$$

when $n$ is greater than $N$. From the convergence of $D_{s}\left(x_{1}\right)$, the relation between $x_{1}$ and $\bar{x}$ in (14), and Theorem VI as applied to $D_{s}\left(x_{1}\right)$, it follows that $\bar{x}$ is in the interior of the region of convergence of $D_{s}(x)$.

2. If we suppose next that $\bar{x}$ is a point in the interior of the region of convergence of $D_{s}(x)$ it may be shown that it is likewise in the interior of the region of convergence of $D(x)$. For this it is obviously sufficient to apply Lemma $I$ in the same way as in the preceding case, taking for $u_{n}$ and $v_{n}$ the values

$$
u_{n}=c_{n} e^{\lambda_{e n} x_{0}^{\natural}}, \quad v_{n}=e^{\lambda_{1 n} x_{1}+\ldots+\lambda_{t n} x_{1} t-\lambda_{\text {en }} x_{0}},
$$

where $x_{0}$ and $x_{1}$ satisfy relation (14), the curve $R\left(e^{i \theta_{*}} x^{8}\right)=\lambda$ being now the boundary of the region of convergence of $D_{s}(x)$.

From the conclusions of the two preceding paragraphs we see that Theorem IX is true in so far as it relates to the region of convergence. The part relating to the region of absolute convergence may be proved in the same way; or it may be proved more directly by a term-by-term comparison of the absolutely convergent series $D\left(x_{0}\right)$ and $D_{s}\left(x_{1}\right)$ in the first case and $D\left(x_{1}\right)$ and $D_{s}\left(x_{0}\right)$ in the second case, $x_{0}$ and $x_{1}$ being connected with an interior point $\bar{x}$ of absolute convergence by a relation of the form (14), the curve $R\left(e^{i \theta_{\star}} \bar{x}^{8}\right)=\lambda$ being now the boundary of the region of absolute convergence.

In the next section we shall have use also for the following somewhat special theorem:

Theorem X. Let $\lambda_{s n}$ be separated into two parts $\rho_{s n}$ and $\sigma_{s n}$ in such a way that $\lambda_{s n}=\rho_{s n}+\sigma_{s n}$ and

$$
\lim _{n=\infty} \frac{\sigma_{s n}}{\rho_{s n}}=0, \quad \lim _{n=\infty} \arg \rho_{s n} \text { exists and is finite. }
$$

Then the boundary curve of the region of convergence [absolute convergence] is the same for the series $D_{s}(x)$ and $\bar{D}_{s}(x)$, where

$$
\bar{D}_{s}(x)=\sum_{n=1}^{\infty} c_{n} e^{\rho_{e n} x^{s}},
$$

provided that $\lambda_{s n}$ has the property (4). 
We observe that the series $\bar{D}_{s}(x)$ is obviously a special case of the series $D(x)$.

The proof of the theorem falls into two parts.

1. Let $\bar{x}$ be any point in the interior of the region of convergence of $D_{s}(x)$. We shall show that $\bar{x}$ is likewise in the interior of the region of convergence of $\bar{D}_{s}(x)$.

If $R\left(e^{i \theta_{s}} x^{s}\right)=\lambda$ is the boundary of the region of convergence of $D_{s}(x)$, then $R\left(e^{i \theta_{0}} \bar{x}^{s}\right)<\lambda$. It is thence obvious that numbers $x_{0}$ and $x_{1}$ exist such that relation (14) is satisfied. Then $x_{0}$ and $x_{1}$ are points of convergence of $D_{s}(x)$. If we apply Lemma I, taking for $u_{n}$ and $v_{n}$ the values

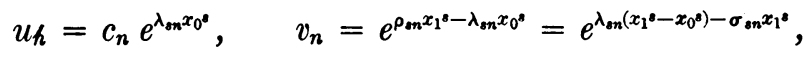

we see that $\bar{D}_{s}\left(x_{1}\right)$ converges if $\sum\left|v_{n+1}-v_{n}\right|$ converges. That the latter series converges follows readily from relation (14), the second form of the value of $v_{n}$, and the fact that $\lambda_{s n}$ has the property (4).

From the convergence of $\bar{D}_{s}\left(x_{1}\right)$, the relation between $x_{1}$ and $\bar{x}$ in (14), and Theorem VI as applied to the series $\bar{D}_{s}(x)$, it follows that $\bar{x}$ is in the interior of the region of convergence of $\bar{D}_{s}(x)$.

2. If $\bar{x}$ is any point in the interior of the region of convergence of $\bar{D}_{s}(x)$ then it may be shown by a method similar to that employed in the previous case that $\bar{x}$ is likewise in the interior of the region of convergence of $D_{s}(x)$.

Thus we have established the part of the theorem which refers to the region of convergence. That referring to the region of absolute convergence may readily be established by the method indicated for the corresponding part of the preceding theorem.

6. Determination of the Regions of Convergence of the SERies $\Omega(x)$ AND $\bar{\Omega}(x)$ IN TERMS OF THEIR COEFFICIENTS

Since the series $\Omega(x)$ and $\bar{\Omega}(x)$ have the same region of convergence and the same region of absolute convergence we shall confine our attention to one of them alone, namely, $\Omega(x)$, in investigating the problem of the determination of the boundary lines of the regions of convergence and absolute convergence in terms of the coefficients $c_{n}$.

An instance $\Delta(x)$ of the series $D(x)$ is intimately related to the series $\Omega(x)$ and is a useful auxiliary in the solution of the problem treated in this section. It may be defined by the relation

$$
\Delta(x)=\sum_{n=1}^{\infty} c_{n} e^{\sigma_{n}} e^{p_{n}(x) \log n+q_{x}(x)-\sigma_{n}} .
$$

where $\sigma_{n}$ has the value 


$$
\sigma_{n}=\nu_{0 n} \log n+\beta_{0 n}
$$

and $p_{n}(x), q_{n}(x), \nu_{0 n}$, and $\beta_{0 n}$ are the functions denoted by these symbols in $\S 2$. It is easy to see that $\Delta(x)$ is that special case of $D(x)$ in which $c_{n}$ is replaced by $c_{n} e^{\sigma_{n}}$ and the relation

$$
\lambda_{1 n} x+\lambda_{2 n} x^{2}+\cdots+\lambda_{l n} x^{t}=p_{n}(x) \log n+q_{n}(x)-\sigma_{n}
$$

is satisfied identically in $x$ for every value of $n$. The last relation determines a set of functions $\lambda_{1 n}, \cdots, \lambda_{t n}$ satisfying the conditions imposed at the beginning of $\S 5$.

The following theorem states an important relation between $\Omega(x)$ and $\Delta(x)$ :

THEOREM XI. Let $x_{0}$ be a non-exceptional point for $\Omega(x)$. Then if either of the series $\Omega(x), \Delta(x)$ converges [converges absolutely] for $x=x_{0}$ the other likewise converges [converges absolutely].

The part of the theorem relating to absolute convergence is obvious in view of the asymptotic relation (7). It remains therefore to prove only the part relating to convergence.

Suppose first that $\Omega\left(x_{0}\right)$ converges. Put

$$
u_{n}=c_{n} g\left(x_{0}+n\right), \quad v_{n}=\frac{e^{p_{n}\left(x_{0}\right) \log n+q_{n}\left(x_{0}\right)}}{g\left(x_{0}+n\right)} .
$$

Then from Lemma $I$ it follows that $\Delta\left(x_{0}\right)$ converges provided that the series $\sum\left|v_{n+1}-v_{n}\right|$ converges. But the convergence of the latter is obvious, since in view of (7) $v_{n}$ is asymptotic with respect to $n$ to an expression proceding in negative integral powers of $n$. Hence $\Delta\left(x_{0}\right)$ converges.

If we suppose that $\Delta\left(x_{0}\right)$ converges we may prove in a similar way that $\Omega\left(x_{0}\right)$ also converges.

Thus Theorem XI is established.

By means of the foregoing theorem we may readily determine the boundary lines of the regions of convergence and absolute convergence of the series $\Omega(x)$. For the discussion we make a separation into two cases according as $k \geqq m$ or $k<m$.

Suppose first that $k \geqq m$. From Theorems XI and IX it follows that the boundary line of the region of convergence [absolute convergence] of $\Omega(x)$ is the same as that of $\Delta(x)$ and hence the same as that of the series

$$
\sum_{n=1}^{\infty} c_{n} e^{\sigma_{n}} e^{\left(\nu_{1 n} \log n+\beta_{1 n}+\gamma_{1 n}\right) x} .
$$

From Theorem $\mathrm{X}$ it follows that the boundary of the region of convergence [absolute convergence] of the latter series, and hence of $\Omega(x)$, is the same as that of the series 


$$
\sum_{n=1}^{\infty} c_{n} e^{\sigma_{n}} e^{(k n n-1 \log n)\left(\mu_{k} x\right)}
$$

In case $k<m$ (and hence $m \geqq 2$ ) it may be shown similarly that the boundary of the region of convergence [absolute convergence] of $\Omega(x)$ is the same as that of the series

$$
\sum_{n=1}^{\infty} c_{n} e^{\sigma_{n}} e^{m n m-1\left(a_{m} x\right)}
$$

Let $\bar{\lambda}$ and $\bar{\mu}$ denote the abscissæ of convergence and absolute convergence respectively of the Dirichlet series

$$
\sum_{n=1}^{\infty} b_{n} e^{-\lambda_{n} x}
$$

where $\lambda_{1}<\lambda_{2}<\lambda_{3}<\cdots$ and $\lambda_{n}$ approaches infinity as $n$ increases indefinitely. Then for the values of $\bar{\lambda}$ and $\bar{\mu}$ we have*

$$
\begin{aligned}
& \bar{\lambda}=\limsup _{\xi=\infty} \frac{\log \left|\sum_{\xi} b_{\nu}\right|}{\xi}, \\
& \mu=\limsup _{\xi=\infty} \frac{\log \sum_{\xi}\left|b_{\nu}\right|}{\xi},
\end{aligned}
$$

where $\xi$ denotes a continuously increasing variable and $\sum_{\xi} \beta_{\nu}$ stands for the sum of all $\beta_{\nu}$ whose suffix $\nu$ satisfies the relation

$$
E(\xi) \leqq \lambda_{\nu}<\xi,
$$

$E(\xi)$ denoting the greatest integer not greater than $\xi$. (If no suffix $\nu$ satisfies this relation then $\sum_{\xi} \beta_{\nu}$ has the value zero.)

Now (18) and (19) are special cases of (20) in which $x$ is replaced by $-\mu_{k} x$ in the first instance and by $-\alpha_{m} x$ in the second instance. The boundary of the region of convergence [absolute convergence] of (20) is

$$
R(x)=\bar{\lambda}[R(x)=\bar{\mu}] .
$$

As in the earlier part of this paper we denote the boundary of the region of

* These values for $\bar{\lambda}$ and $\bar{\mu}$ are due to T. Kojima, To h o k u M a th e mati c a l J o u r $\mathrm{n}$ a l, vol. 6 (1914): 134-139. If $\bar{\lambda}$ is not negative [not positive] we also have

$$
\bar{\lambda}=\limsup _{n=\infty} \frac{\log \left|\sum_{\nu=1}^{n} b_{\nu}\right|}{\lambda_{n}} \quad\left[\bar{\lambda}=\limsup _{n=\infty} \frac{\log \left|\sum_{\nu=n}^{\infty} b_{\nu}\right|}{\lambda_{n}}\right]
$$

and corresponding formulæ for $\bar{\mu}$. The first of the above formulæ for $\bar{\lambda}$ is due to Caben (Annales de l'École Normale Supérièure, ser. 3, vol. 11 (1894): 88-91) and the second to Schnee (G öt inger $\mathrm{Nachrichten}, 1910$, pp. 1-42). They may be used to obtain formulæ alternative to those in Theorem XII, but less satisfactory on the whole. 
convergence [absolute convergence] of $\Omega(x)$, and hence of (18) or (19), by $R(\sigma x)=\lambda[R(\sigma x)=\mu]$ where $\sigma=\mu_{k}$ or $\sigma=\alpha_{m}$ according as $k \geqq m$ or $k<m$. Hence by comparing (18) -and (19) with (20) and making use of the relation (as to the boundary of the region of convergence [absolute convergence]) between $\Omega(x)$ on the one hand and (18) and (19) on the other hand, we see that the convergence [absolute convergence] number for $\Omega(x)$ is

$$
\lambda=-\limsup _{\xi=\infty} \frac{\log \left|\sum_{\xi} c_{\nu} e^{\sigma_{\nu}}\right|}{\xi}\left[\mu=-\limsup _{\xi=\infty} \frac{\log \sum_{\xi}\left|c_{\nu} e^{\sigma_{\nu}}\right|}{\xi}\right]
$$

where $\sum_{\xi} \beta_{\nu}$ stands for the sum of all $\beta_{\nu}$ whose suffix $\nu$ satisfies the condition

$$
E(\xi) \leqq k \nu^{k-1} \log \nu<\xi \text { or } E(\xi) \leqq m \nu^{m-1}<\xi \text {, }
$$

according as $k \geqq m$ or $k<m$. (When no suffix $\nu$ satisfies the given condition $\sum_{\xi} \beta_{\nu}$ has the value 0 .)

We shall now show that a suitable simplification of the foregoing values for $\lambda$ and $\mu$ leads to the following theorem:

ThEOREM XII. Let $\lambda[\mu]$ denote the convergence [absolute convergence] number for the series $\Omega(x)$ or $\bar{\Omega}(x)$ so that the boundary of the region of convergence [absolute convergence] of this series is $R(\sigma x)=\lambda[R(\sigma x)=\mu]$, where $\sigma$ is $\alpha_{m}$ or $\mu_{k}$ according as $m$ is or is not greater than $k$. Then*

(a) in case $k=1$ and $m=0$ or 1 we have

$$
\lambda=-\limsup _{\xi=\infty} \frac{\log \left|\sum_{\xi} c_{\nu} e^{\sigma_{\nu}}\right|}{\xi}, \quad \mu=-\lim _{\xi=\infty} \frac{\log \sum_{\xi}\left|c_{\nu} e^{\sigma_{\nu}}\right|}{\xi},
$$

where $\sum_{\xi} \beta_{\nu}$ stands for the sum of all $\beta_{\nu}$ whose suffix $\nu$ satisfies the relation

$$
e^{E(\xi)} \leqq \nu<e^{\xi}
$$

(b) in case $k>1$ and $k \geqq m$ we have

(c) in case $k<m$ we have

$$
\lambda=\mu=-\lim _{\nu=\infty} \sup \frac{\log \left|c_{\nu} e^{\sigma_{\nu}}\right|}{k \nu^{k-1} \log \nu} ;
$$

$$
\lambda=\mu=-\limsup _{\nu=\infty} \frac{\log \left|c_{\nu} e^{\sigma_{\nu}}\right|}{m \nu^{m-1}} .
$$

The conclusion in case $(a)$ is obtained from (21) in an obvious manner.

In case $(b)$ the relation between $\nu$ and $\xi$ in $\sum_{\xi} \beta_{\nu}$ is determined by the first continued inequality in (22). For a given $\xi$ sufficiently large it is clear that this relation is satisfied by not more than one value of $\nu$. Hence $\lambda$ and $\mu$ are equal, so that we may confine attention to $\lambda$ alone. Moreover, it is clear that the first superior limit in (21) will be obtained if $\xi$ is allowed to approach

${ }^{*}$ Note the alternative form of this theorem indicated in the corollary below. See also the preceding footnote. 
infinity over those values alone for which a corresponding value of $\nu$ exists satisfying the first continued inequality in (22). But for such a value of $\xi$ we may obviously replace $\xi$ by $k \nu^{k-1} \log \nu$,.where $\nu$ has the value corresponding to $\xi$ in virtue of (22), without changing the value of the superior limit in consideration. Hence we are led to the conclusion stated in $(b)$ of the theorem.

The conclusion in case $(c)$ may be proved in the same way as that in case (b) was established.

From equations (7), (8), and (17) it follows that

$$
g(n) \sim e^{\sigma_{n}} s_{n}(1),
$$

the asymptotic relation being valid with respect to $n$ increasing towards $+\infty$. Hence we are led at once to the following corollary of Theorem XII, at least so far as cases $(b)$ and $(c)$ are concerned:

CoRollary. In the conclusion of Theorem XII the quantity $e^{\sigma_{\nu}}$ (wherever it occurs) may be replaced by $g(\nu)$ without destroying the truth of the statements made.

An almost immediate proof of the theorem of this corollary may also be obtained by comparing the series

$$
\sum_{n=0}^{\infty} c_{n} g(n) e^{-\sigma_{n}} g(x+n)
$$

with the series $\Omega(x)$. By means of Lemma I and relation (23) one proves readily that the two series have the same non-exceptional points of convergence.

Let us consider the instance of the series $\bar{\Omega}(x)$ in which $g(x)=e^{x^{2}}$. Then $\sigma=1$ and $\sigma_{n}=n^{2}$. If we replace $c_{n} e^{n^{2}}$ by $a_{n}$ we may write this instance of $\bar{\Omega}(x)$ in the form

$$
\sum_{n=0}^{\infty} a_{n} e^{2 x \cdot n}
$$

Replacing $e^{2 x}$ by $z$ we have an ordinary ascending power series in $z$. Applying conclusion $(c)$ of Theorem XII we find the Cauchy-Hadamard expression

$$
\limsup _{\nu=\infty} \sqrt[v]{\left|a_{\nu}\right|}
$$

for the reciprocal of the radius of convergence of the power series in $z$, so that our theorem is a generalization of the Cauchy-Hadamard theorem for the radius of convergence of a power series.

Let us consider a second special instance of the series $\bar{\Omega}(x)$, namely, that obtained by putting $g(x)=1 / \Gamma(x)$. If we write $(n-1) ! a_{n}$ for $c_{n}$ the series takes essentially the form 


$$
\sum_{n=1}^{\infty} \frac{(n-1) ! a_{n}}{x(x+1)(x+2) \cdots(x+n-1)} .
$$

From case (a) of Theorem XII, as modified by the corollary, we see that the abscissa $\lambda[\mu]$ of convergence [absolute convergence] is given by the relation

$$
\lambda=-\lim _{\xi=\infty} \sup \frac{\log \left|\sum_{\xi} a_{\nu}\right|}{\xi}\left[\mu=-\lim _{\xi=\infty} \sup \frac{\log \sum_{\xi}\left|a_{\nu}\right|}{\xi}\right],
$$

$\nu$ and $\xi$ being related as in the theorem.

The strip between the boundary line of convergence and the boundary line of absolute convergence of the Dirichlet series (20) is known* to have a width at most equal to

$$
\limsup _{\nu=\infty} \frac{\log \nu}{\lambda_{\nu}}
$$

Applying this fact to series (18) and (19) and remembering their relation to the series $\Omega(x)$ we have the following theorem:

TheOREM XIII. The boundary lines of the regions of convergence and absolute convergence of the series $\Omega(x)[\bar{\Omega}(x)]$ coincide unless $k=1$ and $m=0$ or 1 ; and in the latter case the width of the region of conditional convergence is at most $1 / R\left(\mu_{k}\right)$.

From the theory of Dirichlet series and the relation between $\Omega(x)$ and series (18) it follows in the latter case that this strip of conditional convergence may be non-existent or may have its maximum width or may have an intermediate width, examples of Dirichlet series being readily constructed corresponding to each possibility.

7. Further Relations between the SERIEs $\Omega(x)$ AND $\Delta(x)$ When $k=1$ AND $m=0$ OR 1

A particularly important subclass of the series $\Omega(x)$ and $\bar{\Omega}(x)$ is obtained through restricting $g(x)$ by the condition that $k$ shall be unity and $m$ either zero or unity; for this subclass contains the important series to which solutions of linear homogeneous difference equations give rise. For this special subclass the relation between the series $\Omega(x)$ and $\Delta(x)$ is much deeper than is indicated by the theorems of $\S 6$, as we shall now show. For this case we shall denote the series by $\omega(x)$ and $\delta(x)$, so that we have $\dagger$

$$
\omega(x)=\sum_{n=1}^{\infty} c_{n} g(x+n), \quad \delta(x)=\sum_{n=1}^{\infty} c_{n} u(x, n),
$$

\section{where}

* Landau, Handbuch der Lehre von der Verteilung der Primzahlen, p. 734.

t Here we take $c_{0}=0$ as a matter of convenience. 


$$
u(x, n)=n^{\mu_{0}+\mu_{1}(x+n)} e^{a_{0}+\mu_{1} x+\beta(x+n)},
$$

$\beta$ having the value $\alpha_{1}$ or 0 according as $m=1$ or $m=0$. For $g(x+n)$ we have with respect to $n$ the asymptotic relation

$$
g(x+n) \sim u(x, n)\left(1+\frac{h_{1}(x)}{n}+\frac{h_{2}(x)}{n^{2}}+\cdots\right),
$$

the functions $h_{1}(x), h_{2}(x), \cdots$ being polynomials in $x$ which are independent of $n$.

Let us write

$$
\frac{g(x+n)}{u(x, n)}=1+\frac{h_{1}(x)}{n}+\cdots+\frac{h_{\tau}(x)}{n^{\tau}}+\frac{M_{\tau}(x, n)}{n^{\tau+1}},
$$

where $\tau$ is a positive integer. Then $M_{\tau}(x, n)$ approaches a finite value when $x$ is held fixed and $n$ approaches infinity. Thence we have

where

$$
\begin{aligned}
\omega(x)-\delta(x)-h_{1}(x) \delta_{1}(x)-\cdots- & h_{\tau}(x) \delta_{\tau}(x) \\
& =\sum_{n=1}^{\infty} \frac{c_{n}}{n^{\tau+1}} M_{\tau}(x, n) u(x, n),
\end{aligned}
$$

$$
\delta_{i}(x)=\sum_{n=1}^{\infty} c_{n} n^{-i} u(x, n)=e^{-i-\left(\beta i / \mu_{1}\right)} \delta\left(x-i / \mu_{1}\right) \quad(i=1,2, \cdots, \tau) .
$$

Now, let $\lambda$ denote the common convergence number of $\omega(x)$ and $\delta(x)$. Then $\lambda+i$ is the convergence number for the series $\delta_{i}(x)$. Furthermore, it is easy to see also through aid of Theorem XIII that the absolute convergence number, and hence the convergence number, of the series in the second member of (25) is not less than $\lambda+\tau$.

With these results in hand let us consider further the character of the functions $\omega(x)$ and $\delta(x)$ which are defined in an appropriate half-plane by the series $\omega(x)$ and $\delta(x)$ respectively. Let $x_{0}$ be any point such that $\delta(x)$ is analytic at the points

$$
x_{0}-\frac{1}{\mu_{1}}, \quad x_{0}-\frac{2}{\mu_{1}}, \quad x_{0}-\frac{3}{\mu_{1}},
$$

Then the functions $\delta_{i}(x), i=1,2,3, \cdots$, are analytic at the point $x_{0}$. Moreover, only a finite number of the points (26) fail to lie within the common region of convergence of the series $\omega(x)$ and $\delta(x)$. From these considerations and relation (25) it follows readily that the functions $\omega(x)$ and $\delta(x)$ are either both regular or both singular at the point $x_{0}$, provided that $x_{0}$ is non-exceptional for the series $\omega(x)$.

The foregoing results may be gathered into the following theorem: 
Theorem XIV. Let $\omega(x)$ and $\delta(x)$ be functions which are defined in an appropriate half-plane by the series in (24). If $\delta(x)$ is analytic at each of the points (26) and no one of these points is exceptional for the series $\omega(x)$, then the functions $\omega(x)$ and $\delta(x)$ are both regular or both singular at the point $x=x_{0}$.

By means of the last theorem we may readily prove the following:

THEOREM XV. If the numbers

$$
c_{n} n^{\mu_{0}+\mu_{1} n} e^{\beta_{n}},
$$

with the exception at most of a finite number of them, are real and non-negative and if the convergence number $\lambda$ of the series $\omega(x)$ is finite, then the function $\omega(x)$ defined by this series has a singularity at the point $x=\lambda / \mu_{1}$ on the boundary tine of convergence, provided that this point is non-exceptional for the series $\omega(x)$.

The truth of this theorem is an immediate consequence of Theorem XIV and the well-known fact (see Landau's Handbuch, l. c., p. 880) that the function $\delta(x)$ defined by the Dirichlet series in (24) has a singularity at $x=\lambda / \mu_{1}$.

From the corresponding property of the Dirichlet series $\delta(x)$ it follows that the boundary line of convergence of the series $\omega(x)$ does not always pass through a singular point of the function $\omega(x)$ defined by the series.

\section{ON THE UNIQUENESS OF EXPANSIONS IN SERIES $\Omega(x)$ AND $\bar{\Omega}(x)$}

In order that series of a given type shall afford a convenient tool for investigating the properties of functions representable in terms of them it is necessary that a given function shall have not more than one expansion in series of a specified form in that type, the form being sufficiently restricted. That the series $\Omega(x)$ and $\bar{\Omega}(x)$, for a given function $g(x)$, do not always possess this desirable property is shown by the special case in which

$$
g(x)=\Gamma(x)
$$

If in the series $\bar{\Omega}(x)$, for this special case, we replace $x$ by $-z$ the series takes the form

$$
\gamma_{0}+\sum_{n=1}^{\infty} \gamma_{n} z(z-1) \cdots(z-n)
$$

Pincherle* has shown that a series of this form may represent 0 without having all its coefficients equal to zero and that when a function admits an expansion in a series of such form it admits an infinity of such expansions.

The object of this section is to exhibit an extensive subclass of each of the series $\Omega(x)$ and $\bar{\Omega}(x)$ in which the uniqueness expansion theorem is valid

*Attidella Reale Accademia dei Lincei, Rendiconti, ser. 5, vol. 11, pp. 417-426. 
in such wise that no function has more than one expansion in a series $\Omega(x)$ or a series $\bar{\Omega}(x)$ for a given function $g(x)$ of the type through which these subclasses arise.

As before we employ $\sigma$ to denote $\alpha_{m}$ or $\mu_{k}$ according as $m$ is or is not greater than $k$. We have seen that the points in the interior of the region of convergence of a series $\Omega(x)$ or a series $\bar{\Omega}(x)$ are all the points $x$ satisfying the condition $R(\sigma x)<\lambda$ where $\lambda$ is an appropriately determined constant. If the real part of $\sigma$ is negative and $x$ is any fixed number then it is clear that only a finite number (at most) of the points $x, x+1, x+2, \cdots$ fail to lie within the region of convergence of the series; but if $R(\sigma)$ is positive an infinite number of these points lie outside of the region ot convergence. Moreover, if $R(\sigma)$ is negative it is clear that $g(x+n)$ approaches zero as $n$ increases indefinitely. These considerations taken in connection with the form of $\Omega(x)$ and $\bar{\Omega}(x)$ direct attention to the case in which $R\left(\sigma^{\prime}\right)$ is negative. We shall now show that the desired uniqueness theorem is true in case this condition is satisfied.

As a preliminary to this end let us consider the positive real-valued function $h(u)$ of the real variable $u$ defined by the relation

where

$$
h(u)=u^{p(u)} e^{q(u)},
$$

$$
\begin{aligned}
& p(u)=\nu_{0}+\nu_{1} u+\nu_{2} u^{2}+\cdots+\nu_{k} u^{k}, \\
& q(u)=\beta_{0}+\beta_{1} u+\beta_{2} u^{2}+\cdots+\beta_{m} u^{m},
\end{aligned}
$$

the constants $\nu$ and $\beta$ having the values

$$
\nu_{j}=R\left(\mu_{j}\right), \quad j=0,1, \cdots, k ; \quad \beta_{j}=R\left(\alpha_{j}\right), \quad j=0,1, \cdots, m .
$$

Since $R(\sigma)$ is negative we have $\nu_{k}$ negative or $\beta_{m}$ negative according as $k \geqq m$ or $k<m$. Thence it is easy to see that the ratio $h(u+1) / h(u)$ constantly decreases as $u$ increases, provided at least that $u$ is sufficiently large.

Now, let us suppose that a given function $f(x)$ has the expansions

$$
f(x)=\sum_{n=0}^{\infty} c_{n} g(x+n)=\sum_{n=0}^{\infty} d_{n} g(x+n),
$$

both expansions being valid for $R(\sigma x)$ less than some number $\lambda_{1}$. We shall show that $c_{n}=d_{n}$ for every value of $n$. For this purpose it is obviously sufficient to show that if the relation

$$
\sum_{n=0}^{\infty} \gamma_{n} g(x+n)=0
$$


is valid for every non-exceptional value of $x$ for which $R(\sigma x)<\lambda_{1}$ then $\gamma_{0}=0=\gamma_{1}=\gamma_{2}=\cdots$. From equation (28) and the asymptotic character of the function $g(x)$ and the relation between $g(x)$ and $h(u)$ we see that

$$
\left|\gamma_{0}\right| \leqq \sum_{n=0}^{\infty}\left|\gamma_{n}\right|\left|\frac{g(u+n)}{g(u)}\right|<M \frac{h(u+1)}{h(u)} \sum_{n=1}^{\infty}\left|\gamma_{n}\right| \frac{h(u+n)}{h(u+1)},
$$

$M$ being an appropriately determined constant. That the series in the last member of this relation converges when $u$ is sufficiently large follows at once from the fact that its absolute convergence number is the same as that of the series $\Delta(x)$ associated with the series $\Omega(x)$ in equation (28). We shall show that the sum of this series is bounded as $u$ increases. It may be written in the form

$$
\sum_{n=1}^{\infty}\left|\gamma_{n}\right| \frac{h(u+2)}{h(u+1)} \cdot \frac{h(u+3)}{h(u+2)} \cdots \cdots \frac{h(u+n)}{h(u+n-1)} .
$$

Now, when $u$ is sufficiently large each of the fractions $h(u+i+1) / h(u+i)$ decreases as $u$ increases; and hence the sum of this series does not increase indefinitely as $u$ increases. Therefore from (28) we have a relation of the form

$$
\left|\gamma_{0}\right|<\bar{M} \frac{h(u+1)}{h(u)},
$$

where $\bar{M}$ is a constant. Letting $u$ increase indefinitely we see from this that $\gamma_{0}=0$. If in (28) we replace $\gamma_{0}$ by 0 and $x$ by $x-1$ we may proceed as before to prove that $\gamma_{1}=0$. Likewise we have in order

$$
\gamma_{2}=0=\gamma_{3}=\gamma_{4}=\cdots \text {. }
$$

Thus we are led to the following theorem:

Theorem XVI. Let $\sigma$ denote $\alpha_{m}$ or $\mu_{k}$ according as $m$ is or is not greater than $k$. Let $g(x)$ be a function for which $R(\sigma)$ is negative. Then a given function $f(x)$ has not more than one expansion in a series $\Omega(x)$ or a series $\bar{\Omega}(x)$ for this given function $g(x)$.

In case a given function $f(x)$ has an expansion in the form

$$
f(x)=\sum_{n=0}^{\infty} c_{n} g(x+n)
$$

and $R(\sigma)$ is negative, the coefficients $c_{0}, c_{1}, \cdots$ may readily be determined by means of the properties of the function $f(x)$ itself. In fact, in view of the argument by means of which the foregoing theorem was proved it is easy to see that we have 


$$
\begin{aligned}
& c_{0}=\lim _{n=+\infty} \frac{f(n)}{g(n)}, \\
& c_{1}=\lim _{n=+\infty} \frac{f(n)-c_{0} g(n)}{g(n+1)}, \\
& c_{2}=\lim _{n=+\infty} \frac{f(n)-c_{0} g(n)-c_{1} g(n+1)}{g(n+2)},
\end{aligned}
$$

In case a given function $f(x)$ has an expansion in the form of a series $\bar{\Omega}(x)$ and $R(\sigma)$ is negative, the coefficients in the expansion may obviously be determined in a similar manner.

UNIVERSITY OF ILLINOIS,

October, 1915 